Contents List available at RAZI Publishing

Matriks Sains Matematik (MSMK)

Journal Homepage: http://www.razipublishing.com/journals/matriks-sains-matematik

https://doi.org/10.26480/msmk.02.2017.17.21

\title{
ON APPLICATIONS OF COUPLED FIXED-POINT THEOREM IN HYBRID DIFFERENTIAL EQUATIONS OF ARBITRARY ORDER
}

\author{
Muhammad Shaob, Kamal Shah*, Rahmat Ali Khan \\ Department of Mathematics, University of Malakand, Khyber Pakhtunkhwa, Pakistan.
} *Corresponding Author email: kamalshah408@gmail.com

This is an open access article distributed under the Creative Commons Attribution License, which permits unrestricted use, distribution, and reproduction in any medium, provided the original work is properly cited

\section{ARTICLE DETAILS}

\section{ARTICLE HISTORY:}

Received 6 July 2017

Accepted 10 October 2017

Available online 5 November 2017

\section{KEYWORDS:}

Coupled systems, Boundary value problems, Fractional differential equations, Existence results, Hybrid fixed point theorems.

\section{ABSTRACT}

In this manuscript, we use fixed point theorem due to Bashiri theory and develop sufficient conditions for existence of solution of coupled system of fractional differential equation in Banach space.

\section{INTRODUCTION AND PRELIMINARIES}

Currently in most of the research areas the rate of fractional differential equations has been increased due to wide range of application of fractional calculus theory in the problems of real life. In various scientific and engineering disciplines such as physics, mechanics, chemistry these applications can be found. It can be also used in control theory, optimization theory, signal processing, economics etc. [1-5]. Beside this, due to its existence in daily life situations, most of the authors are motivated towards the existence and uniqueness of solutions of fractional differential equations $[6,7,9,10$,$] .$

Using different types of fixed point theorems such as Banach contraction principle, Schaefer fixed point theorem, and LeraySchauder degree are studied in detail for existence and uniqueness of solutions to multi-points boundary value problems $[13,15-17,19,20]$.

Dhage and Lakshmikantham studied the existence and uniqueness theorems of the solution to the ordinary first-order hybrid differential equation with perturbation of first type

$$
\frac{d}{d x}\left[\frac{x(t)}{f(t, x(t))}\right]=g(t, x(t)) \text { a.e } t \in J, x\left(t_{0}\right)=x_{0} \in \mathrm{R},
$$

where $f \in C(J \times \mathrm{R}, \mathrm{R}-\{0\})$ and $g \in \zeta(J \times \mathrm{R}, \mathrm{R})$, where $J=\left[t_{0}, t_{0}+a\right]$ is bounded interval in $\mathrm{R}$ for some $t_{0}$ and $a \in \mathrm{R}$ with $a>0, C(J \times \mathrm{R}, \mathrm{R})$ is the class of continues function and $\zeta(J \times \mathrm{R}, \mathrm{R})$ is called the

caratheodory class of function $g: J \times \mathrm{R} \rightarrow \mathrm{R}$ which are Lebegue integrable bounded by a Lebesgue integrable function on $J$ [5] Furthermore

i. the map $t \rightarrow g(t, x)$ is measurable for each $x \in \mathrm{R}$;

ii. the map $t \rightarrow g(t, x)$ is continues for each $t \in J$.
Some of study studied the existence and uniqueness theorems of the solution of the ordinary first-order hybrid differential equation with perturbation of second type [36].

$$
\frac{d}{d x}[x(t)-f(t, x(t))]=g(t, x(t)) \text { a.et } t \in J, x\left(t_{0}\right)=x_{0} \in \mathrm{R} .
$$

Ammiet et al. [37], focus on the generalization of (1.1) by replacing the ordinary by fractional derivative in Riemann-Liouville sense. Furthermore, a researcher generalizes (1.2) by replacing the classical differentiation by fractional derivative in the Riemann-Liouville sense [38].

In other research, author discuss the existence of solutions to hybrid fractional differential equations in both types using the Caputo's fractional derivative instead of the classical one in both (1.1) and (1.2) [8].

Recently, existence of solutions to boundary value problems for coupled systems of fractional order differential equations have also attracted some attentions, we refer to $[11,12,14,40]$.

The hybrid differential equations have been much more attractive, see, so there have been many works on the theory of hybrid differential equations [18, 39]. Additionally, hybrid fixed point theory can be used to develop the existence theory for the hybrid equations $[2,4,6,15-21]$.

Lately, researcher discussed a two-point boundary value problem for a coupled system of fractional differential equations [12]. There also some researcher analyzed the solutions of coupled nonlinear fractional reaction-diffusion equations [40]

Motivated with the above works, our purpose in this paper is to prove the existence of solution to the following system of fractional hybrid differential equations of order for $n-1<\alpha \leq n$

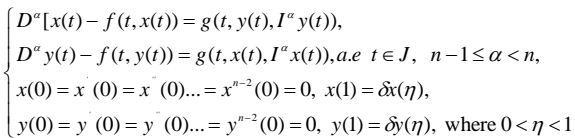


Definition 1.1 The form of Riemann-Liouville fractional integral operator of order $\alpha>0$ of function $f \in L^{1}\left(\mathrm{R}^{+}\right)$is defined as

$$
I^{\alpha} f(t)=\frac{1}{\Gamma(\alpha)} \int_{0}^{t}(t-s)^{\alpha-1} f(s) d s .
$$

Definition 1.2 Let \& be a positive real number, such that $m-1 \leq \alpha<m$ , $m \in \mathrm{N}$ and $f^{m}(x)$ exists a function of class $C$. Then Caputo's fractional derivative of $f$ is defined as

$$
D^{\alpha} f(t)=\frac{1}{\Gamma(m-\alpha)} \int_{0}^{t}(t-s)^{m-\alpha-1} f^{m}(s) d s
$$

Definition 1.3 The Riemann-Liouville fractional integral operator of order $\alpha>0$ of continuous function $f: \mathrm{R}^{+} \rightarrow \mathrm{R} f \in L^{1}\left(R^{+}\right)$is defined as

$$
D^{\alpha} f(t)=\frac{1}{\Gamma(n-\alpha)} \int_{0}^{t}(t-s)^{n-\alpha-1} f(s) d s .
$$

Lemma 1.4 Let $0<\alpha<1$ and $f \in L^{1}(0,1)$. Then

$$
D^{\alpha} I^{\alpha} f(t)=f(t)
$$

hold.

$$
I^{\alpha} D^{\alpha} f(t)=f(t)-\frac{\left[D^{\alpha-1} f(t)\right]_{t=0}}{\Gamma(\alpha)} t^{\alpha-1} .
$$

holds almost everywhere on $J$

Lemma 1.5 [28]. The fractional differential equation of order $q>0$

$$
{ }^{c} D^{q} y(t)=0, n-1 \leq<n,
$$

has a unique solution of the form?

$$
y(t)=c_{0}+c_{1} t+c_{2} t^{2}+\ldots+c_{n-1} t^{n-1},
$$

where $c_{i} \in \mathrm{R}, i=0,1,2, \ldots, n-1$, such that $n=[q]+1$.

Lemma 1.6 [28]. The following result holds for fractional differential equations

$$
I^{q^{c}} D^{q} y(t)=y(t)+c_{0}+c_{1} t+c_{2} t^{2}+\ldots+c_{n-1} t^{n-1}
$$

for arbitrary $c_{i} \in \mathrm{R}, i=0,1,2, \ldots, n-1$, such that $n=[q]+1$.

Theorem 1.7 [2]. Let $W \neq \phi$, closed, convex and bounded subset of the Banach space $X$ and $\tilde{S}=S \times S$. Let $A: X \rightarrow X$ and $B: S \rightarrow X$ be two operators such that

i. $A$ is contraction with constant alpha $<1$;

ii. $B$ is completely continuous;

iii. $x=A x+B y$ implies for all $y \in S \Rightarrow x \in S$, and

then the operator equations $T(x, y)=A x+B y$ has at least a coupled fixed point in $\tilde{S}$ whenever $\sigma<1$.

Theorem 1.8 [2]. Let $W \neq \phi$, closed, convex and bounded subset of the Banach space $X$ and $\tilde{S}=S \times S$. Let $A: X \rightarrow X$ and $B: S \rightarrow X$ be two operators such that

i. There exist $\phi_{A} \in \Phi$ such that for all $x, y \in X$, we have; $\|A x-A y\| \leq \sigma \phi_{A}(\|x-y\|)$;

ii. $B$ is completely continuous;

iii. $x=A x+B y$ implies for all $y \in S \Rightarrow x \in S$, and

then the operator equations $T(x, y)=A x+B y$ has at least a coupled fixed point in $\tilde{S}$ whenever $\sigma<1$.

Now, Consider the following assumption

$\left(\mathrm{C}_{0}\right)$ The function $x \rightarrow x-f(t, x)$ is increasing in $\mathrm{R}$ for all $t \in J$;
(C1) There exist a constant $M \geq L>0$, such that

$\left.\mid f(t, x(t))-f(t, y(t)) \leq \frac{L|x(t)-y(t)|}{8(M+|x(t)-y(t)|)}\right) ;$

$\left(\mathrm{C}_{2}\right) \frac{L \delta|x(\eta)-y(\eta)|}{8 \Delta(M+\delta|x(\eta)-y(\eta)|)} \leq \frac{L \delta|x(t)-y(t)|}{8(M+\delta|x(t)-y(t)|)} \leq L$

$\frac{L|x(\eta)-y(\eta)|}{8 \Delta(M+|x(\eta)-y(\eta)|)} \leq \frac{L|x(t)-y(t)|}{8(M+|x(t)-y(t)|)} \leq L$

$\left(\mathrm{C}_{3}\right)$ Fix

$$
F_{0}=\max _{t \in J}|f(t, 0)|
$$

$\left(\mathrm{C}_{4}\right)$ There exist a continuous function constant $h \in C(J, \mathrm{R})$ such that $g(t, x(t), y(t)) \leq h(t), t \in J, x, y \in \mathrm{R}$

Lemma 1.9 Assume that hypothesis $\left(C_{0}\right)$ holds. Then, for any $y \in C(J, \mathrm{R})$ and $0<\alpha<1$, the function $f \in C(J, \mathrm{R})$ with $f(0,0)=0$ and $\left.\frac{\partial^{i} f(t, x(t))}{a^{t}}\right|_{t=0}=0, i=1,2 \ldots . . n-2$. Then the unique solution of the FHDE

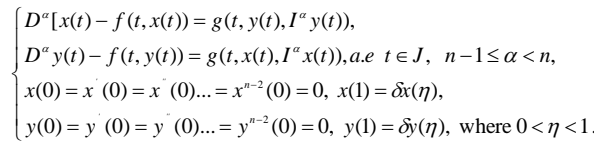

is

$$
\begin{aligned}
& \left\{\begin{array}{l}
x(t)=f(t, x(t))+t^{n-1}\left[\frac{\delta[f(\eta, x(\eta))-f(1, x(1))}{\left(1-\eta^{n-1}\right)}\right]+I^{\alpha} g\left(t, y(t), I^{\alpha} y(t)\right) \\
+t^{n-1}\left[\frac{\delta\left[I^{\alpha} g\left(\eta, y(\eta), I^{\alpha}\right) y(\eta)\right]-I^{\alpha} g\left(1, y(1), I^{\alpha} y(1)\right)}{\left(1-\eta^{n-1}\right)}\right] .
\end{array}\right. \\
& \left\{\begin{array}{l}
y(t)=f(t, y(t))+t^{n-1}\left[\frac{\delta[f(\eta, y(\eta))-f(1, y(1))}{\left(1-\eta^{n-1}\right)}\right]+I^{\alpha} g\left(t, x(t), I^{\alpha} x(t)\right) \\
+t^{n-1}\left[\frac{\delta\left[I^{\alpha} g\left(\eta, x(\eta), I^{\alpha}\right) x(\eta)\right]-I^{\alpha} g\left(1, x(1), I^{\alpha} x(1)\right)}{\left(1-\eta^{n-1}\right)}\right] .
\end{array}\right.
\end{aligned}
$$

Proof Let $x$ be a solution of the Cauchy problem. Since the RiemannLiouville fractional integral $I^{q}$ is a monotone operator, thus we apply the fractional integral $I^{q}$ on both sides of

$$
I^{\alpha}\left[{ }^{c} D^{\alpha} u(t)\right]=u(t)+C_{0}+C_{1} t+C_{2} t^{2}+\ldots+C_{n-1} t^{n-1},
$$

where $n=[\alpha]+1$ and $C_{i} \in \mathrm{R}^{+}$and $I^{\alpha}$ is integral operator of fractional order.

$$
\left\{\begin{array}{l}
I^{\alpha}\left[{ }^{c} D^{\alpha}[x(t)-f(t, x(t))]=I^{\alpha}\left[g\left(t, y(t), I^{\alpha}\right) y(t)\right],\right. \\
x(t)-f(t, x(t))=I^{\alpha} g\left(t, y(t), I^{\alpha} y(t)\right)+C_{0}+C_{1} t+C_{2} t^{2}+\ldots+C_{n-1} t^{n-1}, \\
x(t)=f(t, x(t))+I^{\alpha} g\left(t, y(t), I^{\alpha} y(t)\right)+C_{0}+C_{1} t+C_{2} t^{2}+\ldots+C_{n-1} t^{n-1}, \\
x(t)=f_{t}(t, x(t))+I^{\alpha-1} g\left(t, y(t), I^{\alpha} y(t)\right)+C_{1}+2 C_{2} t+3 C_{3} t^{2}+\ldots+C_{n-1} t^{n-2}, \\
x(t)=f_{n t}(t, x(t))+I^{\alpha-2} g\left(t, y(t), I^{\alpha} y(t)\right)+2 C_{2}+3.2 C_{3} t+\ldots+C_{n-1} t^{n-2}, \\
\vdots \\
x^{n-2}(t)=f_{n-2}(t, x(t))+I^{\alpha-(n-2)} g\left(t, y(t), I^{\alpha} y(t)\right)+(n-2)(n-3) \ldots 2 . C_{n-2} \\
+(n-1)(n-2) \ldots 2 C_{n-1} t,
\end{array}\right.
$$

If we assume $f(0,0)=0$, then $c_{0}=0$ from 2 nd equation of above system. If assume $\left.\frac{\partial f(t, x(t))}{\partial t}\right|_{t=0}=0$, then $c_{1}=0$ from 3rd equation of above system. Similarly, in this way in last if assume $\left.\frac{\partial^{n-2} f(t, x(t))}{\partial t^{n-2}}\right|_{t=0}=0$, then $c_{n-2}=0$.

$$
\begin{gathered}
x(t)=f(t, x(t))+I^{\alpha} g\left(t, y(t), I^{\alpha} y(t)\right)+C_{n-1} t^{n-1}, \\
x(1)=f(1, x(1))+I^{\alpha} g\left(1, y(1), I^{\alpha} y(t)\right)+C_{n-1}, \\
\delta x(\eta)=\delta\left[f(\eta, x(\eta))+I^{\alpha} g\left(\eta, y(\eta), I^{\alpha} y(\eta)\right)+C_{n-1} \eta^{n-1},\right. \\
\left(1-\eta^{n-1}\right) C_{n-1}=-f(1, x(1))-I^{\alpha} g\left(1, y(1), I^{\alpha} y(1)\right) \\
+\delta\left[f(\eta, x(\eta))+I^{\alpha} g\left(\eta, y(\eta), I^{\alpha} y(\eta)\right),\right. \\
C_{n-1}=\frac{-f(1, x(1))-I^{\alpha} g\left(1, y(1), I^{\alpha}\right)+\delta\left[f(\eta, x(\eta))+I^{\alpha} g\left(\eta, y(\eta), I^{\alpha} y(\eta)\right)\right.}{\left(1-\eta^{n-1}\right)}, \\
x(t)=f(t, x(t))+I^{\alpha} g\left(t, y(t), I^{\alpha} y(t)\right) \\
+t^{n-1}\left[\frac{-f(1, x(1))-I^{\alpha} g\left(1, y(1), I^{\alpha} y(1)\right)+\delta\left[f(\eta, x(\eta))+I^{\alpha} g\left(\eta, y(\eta), I^{\alpha} y(\eta)\right)\right.}{\left(1-\eta^{n-1}\right)}\right],
\end{gathered}
$$

Similarly, by the same procedure, we get 


$$
\begin{gathered}
y(t)=f(t, y(t))+I^{\alpha} g\left(t, x(t), I^{\alpha} x(t)\right) \\
+t^{n-1}\left[\frac{-f(1, y(1))-I^{\alpha} g\left(1, x(1), I^{\alpha} x(1)\right)+\delta\left[f(\eta, y(\eta))+I^{\alpha} g\left(\eta, x(\eta), I^{\alpha} x(\eta)\right)\right.}{\left(1-\eta^{n-1}\right)}\right],
\end{gathered}
$$

By rearranging the terms, we have

$$
\begin{aligned}
& \left\{\begin{array}{l}
x(t)=f(t, x(t))+t^{n-1}\left[\frac{\delta[f(\eta, x(\eta))-f(1, x(1))}{\left(1-\eta^{n-1}\right)}\right]+I^{\alpha} g\left(t, y(t), I^{\alpha} y(t)\right) \\
+t^{n-1}\left[\frac{\delta\left[I^{\alpha} g\left(\eta, y(\eta), I^{\alpha}\right) y(\eta)\right]-I^{\alpha} g\left(1, y(1), I^{\alpha} y(1)\right)}{\left(1-\eta^{n-1}\right)}\right] .
\end{array}\right. \\
& \left\{\begin{array}{l}
y(t)=f(t, y(t))+t^{n-1}\left[\frac{\delta[f(\eta, y(\eta))-f(1, y(1))}{\left(1-\eta^{n-1}\right)}\right]+I^{\alpha} g\left(t, x(t), I^{\alpha} x(t)\right) \\
+t^{n-1}\left[\frac{\delta\left[I^{\alpha} g\left(\eta, x(\eta), I^{\alpha}\right) x(\eta)\right]-I^{\alpha} g\left(1, x(1), I^{\alpha} x(1)\right)}{\left(1-\eta^{n-1}\right)}\right] .
\end{array}\right.
\end{aligned}
$$

Theorem 1.10 Suppose the assumption $\left(C_{0}\right)-\left(C_{4}\right)$ hold. Then the FHDE of system (1) has a solution defined on $J$.

Proof. Let $X=C(J, \mathrm{R})$ and a subset of $X$ defined as

$$
S=\{x \in X,\|x\| \leq N\}
$$

where $2 L+F_{0}+\frac{2\|h\|}{\Gamma(\alpha)}+\frac{\delta\|h\|}{\Gamma(\alpha)}+\frac{\delta L}{2} \leq N$.

Clearly $S$ is bounded, closed and convex subset of the Banach space X. Consider the following systems

$$
\begin{aligned}
& \left\{\begin{array}{l}
x(t)=f(t, x(t))+t^{n-1}\left[\frac{\delta[f(\eta, x(\eta))-f(1, x(1))}{\left(1-\eta^{n-1}\right)}\right]+I^{\alpha} g\left(t, y(t), I^{\alpha} y(t)\right) \\
+t^{n-1}\left[\frac{\delta\left[I^{\alpha} g\left(\eta, y(\eta), I^{\alpha}\right) y(\eta)\right]-I^{\alpha} g\left(1, y(1), I^{\alpha} y(1)\right)}{\left(1-\eta^{n-1}\right)}\right] .
\end{array}\right. \\
& \left\{\begin{array}{l}
y(t)=f(t, y(t))+t^{n-1}\left[\frac{\delta[f(\eta, y(\eta))-f(1, y(1))}{\left(1-\eta^{n-1}\right)}\right]+I^{\alpha} g\left(t, x(t), I^{\alpha} x(t)\right) \\
+t^{n-1}\left[\frac{\delta\left[I^{\alpha} g\left(\eta, x(\eta), I^{\alpha}\right) x(\eta)\right]-I^{\alpha} g\left(1, x(1), I^{\alpha} x(1)\right)}{\left(1-\eta^{n-1}\right)}\right] .
\end{array}\right.
\end{aligned}
$$

Define two operators $A: X \rightarrow X$ and $B: S \rightarrow X$ as

$$
\left\{\begin{array}{l}
A x(t)=f(t, x(t))+t^{n-1}\left[\frac{\delta[f(\eta, x(\eta))-f(1, x(1))}{\left(1-\eta^{n-1}\right)}\right] \\
B x(t)=I^{\alpha} g\left(t, x(t), I^{\alpha} x(t)\right)+t^{n-1}\left[\frac{\delta\left[I^{\alpha} g\left(\eta, x(\eta), I^{\alpha}\right) x(\eta)\right]-I^{\alpha} g\left(1, x(1), I^{\alpha} x(1)\right)}{\left(1-\eta^{n-1}\right)}\right] .
\end{array}\right.
$$

Systems (1.12) and (1.13) can be written as

$$
\left\{\begin{array}{l}
x(t)=A x(t)+B y(t), \\
y(t)=A y(t)+B x(t)
\end{array}\right.
$$

Now, we want to check all condition of Theorem (1.18) or operators $A$ , $B$, we have

Let $x, y \in X$ by assumption $C_{1}$, we obtain

$$
\begin{gathered}
|A x(t)-A y(t)| \mid f(t, x(t))+t^{n-1}\left[\frac{\delta[f(\eta, x(\eta))-f(1, x(1))}{\left(1-\eta^{n-1}\right)}\right]-f(t, y(t)) \\
-t^{n-1}\left[\frac{\delta[f(\eta, y(\eta))-f(1, y(1))}{\left(1-\eta^{n-1}\right)}\right] .
\end{gathered}
$$

Let $\Delta=1-\eta^{n-1}$, then

$|A x(t)-A y(t)| \triangleleft f(t, x(t))-f(t, y(t)) \mid$

$$
+\left|\frac{\delta f(\eta, x(\eta))-f(1, x(1)-\delta(f(\eta, y(\eta))+f(1, y(1))])}{\Delta}\right|,
$$

by assumption, we have

$|A x(t)-A y(t)| \leqslant|f(t, x(t))-f(t, y(t))+| \frac{f(1, y(1))-f(1, x(1))}{\Delta}|+| \frac{\delta[f(\eta, x(\eta))-f(\eta, y(\eta))]}{\Delta} \mid$

$$
\begin{aligned}
& \leq \frac{L|x(t)-y(t)|}{8(M+|x(t)-y(t)|)}+\frac{L \delta|x(\eta)-y(\eta)|}{\Delta 8(M+\delta|x(\eta)-y(\eta)|}+\frac{L \cdot|x(\eta)-y(\eta)|}{\Delta 8(M+|x(\eta)-y(\eta)|} \\
& \leq \frac{L \cdot|x(t)-y(t)|}{8(M+|x(t)-y(t)|)}+\frac{L \cdot|x(t)-y(t)|}{8(M+|x(t)-y(t)|)}+\frac{L \delta|x(t)-y(t)|}{8(M+\delta|x(t)-y(t)|} \\
& =\frac{L \cdot|x(t)-y(t)|}{4(M+|x(t)-y(t)|}+\frac{L \delta|x(t)-y(t)|}{8(M+\delta|x(t)-y(t)|} \\
& =\frac{1}{2}\left(\frac{L .|x(t)-y(t)|}{2(M+|x(t)-y(t)|}+\frac{L \cdot \delta|x(t)-y(t)|}{4(M+\delta|x(t)-y(t)|)}\right) .
\end{aligned}
$$

Which implies that $A$ is a nonlinear contraction on $X$ with control function $\frac{\phi}{2}$ where $\phi$ is defined by

$$
\phi(t)=\frac{L . t}{2(M+t)}+\frac{L . \delta t}{4(M+\delta t)} .
$$

For continuity of $B$, let $\left(x_{m}\right)$ be a sequence in $S$ converging to a point $x \in S$. By Lebesgue Dominated convergence theorem, we have

$$
\begin{aligned}
\lim _{m \rightarrow \infty} B x_{m}(t) & \left.=\frac{1}{\Gamma(\alpha)} \lim _{m \rightarrow \infty} \int_{0}^{t}(t-s)^{\alpha-1} g\left(t, x_{m}(t), I^{\alpha} x_{m}(t)\right)\right) d s \\
& +t^{n-1}\left[\frac{-I^{\alpha} g\left(1, x(1), I^{\alpha} x(1)\right)+\delta\left[I^{\alpha} g\left(\eta, x(\eta), I^{\alpha} x(\eta)\right)\right]}{\left(1-\eta^{n-1}\right)}\right] \\
& \left.=\frac{1}{\Gamma(\alpha)} \int_{0}^{t}(t-s)^{\alpha-1} \lim _{m \rightarrow \infty} g\left(t, x_{m}(t), I^{\alpha} x_{m}(t)\right)\right) d s \\
& +t^{n-1}\left[\frac{-I^{\alpha} g\left(1, \delta x(\eta), I^{\alpha} \delta x(\eta)\right)+\delta\left[I^{\alpha} g\left(\eta, x(\eta), I^{\alpha} x(\eta)\right)\right]}{\left(1-\eta^{n-1}\right)}\right]=B x(t) .
\end{aligned}
$$

Now for boundedness, we have

$$
\begin{aligned}
|B x(t)| & \left.=\frac{1}{\Gamma(\alpha)} \mid \int_{0}^{t}(t-s)^{\alpha-1} g\left(t, x(t), I^{\alpha} x(t)\right)\right) d s \\
& +t^{n-1}\left[\frac{\delta\left[I^{\alpha} g\left(\eta, x(\eta), I^{\alpha} x(\eta)\right)\right]-I^{\alpha} g\left(1, x(1), I^{\alpha} x(1)\right)}{\left(1-\eta^{n-1}\right)}\right] \mid \\
& \left.\leq \frac{1}{\Gamma(\alpha)} \int_{0}^{t}\left|(t-s)^{\alpha-1}\right| g\left(t, x(t), I^{\alpha} x(t)\right)\right) \mid d s \\
& +\left|t^{n-1}\right|\left|\frac{\delta\left[I^{\alpha} g\left(\eta, x(\eta), I^{\alpha} x(\eta)\right)\right]-I^{\alpha} g\left(1, x(1), I^{\alpha} x(1)\right)}{\left(1-\eta^{n-1}\right)}\right| \\
& \left.\left.\leq \frac{1}{\Gamma(\alpha)} \int_{0}^{t}\left|(t-s)^{\alpha-1}\right| h(t)\right)\right)|d s+| t^{n-1}\left|\frac{\delta\left[I^{\alpha} h(\eta)\right]-I^{\alpha} h(1)}{\left(1-\eta^{n-1}\right)}\right| \\
& \leq \frac{1}{\Gamma(\alpha+1)}\|h(t)\|+\left|\frac{\delta\left[I^{\alpha} h(\eta)\right]-I^{\alpha} h(1)}{\left(1-\eta^{n-1}\right)}\right|, \text { using } t \leq 1,
\end{aligned}
$$

for all $x \in S$. So $B$ is uniformly bounded on $S$. Now to prove $B$ is completely continuous let $t_{1}, t_{2} \in J$, such that $t_{1}<t_{2}$ for any $x \in S$, we have

$$
\begin{aligned}
B x\left(t_{1}\right)-B x\left(t_{2}\right) & \left.=\frac{1}{\Gamma(\alpha)} \int_{0}^{t_{1}}\left(t_{1}-s\right)^{\alpha-1} g\left(s, x(t), I^{\alpha} x(t)\right)\right) d s \\
+ & t^{n-1}\left[\frac{-I^{\alpha} g\left(1, x(1), I^{\alpha} x(1)\right)+\delta\left[I^{\alpha} g\left(\eta, x(\eta), I^{\alpha} x(\eta)\right)\right]}{\left(1-\eta^{n-1}\right)}\right] \\
& \left.-\int_{0}^{t_{2}}\left(t_{2}-s\right)^{\alpha-1} g\left(s, x(t), I^{\alpha} x(t)\right)\right) d s-t^{n-1}\left[\frac{I^{\alpha} g\left(1, x(1), I^{\alpha} x(1)\right)+\delta\left[I^{\alpha} g\left(\eta, x(\eta), I^{\alpha} x(\eta)\right)\right]}{\left(1-\eta^{n-1}\right)}\right] \\
& \left.\left.\leq \frac{1}{\Gamma(\alpha)} \int_{0}^{t_{1}}\left(t_{1}-s\right)^{\alpha-1} g\left(s, x(t), I^{\alpha} x(t)\right)\right) d s-\int_{0}^{t_{1}}\left(t_{2}-s\right)^{\alpha-1} g\left(s, x(t), I^{\alpha} x(t)\right)\right) d s \\
& +t^{n-1}\left[\frac{-I^{\alpha} g\left(1, x(1), I^{\alpha} x(1)\right)-I^{\alpha} g\left(1, x(1), I^{\alpha} x(1)\right)}{\left(1-\eta^{n-1}\right)}\right] \\
& +t^{n-1}\left[\frac{\delta\left[I^{\alpha} g\left(\eta, x(\eta), I^{\alpha} x(\eta)\right)-\delta\left[I^{\alpha} g\left(\eta, x(\eta), I^{\alpha} x(\eta)\right)\right]\right.}{\left(1-\eta^{n-1}\right)}\right] \\
& \left.\left.\leq \frac{1}{\Gamma(\alpha)} \int_{0}^{t_{1}}\left(t_{1}-s\right)^{\alpha-1} g\left(s, x(t), I^{\alpha} x(t)\right)\right) d s-\int_{0}^{t_{2}}\left(t_{2}-s\right)^{\alpha-1} g\left(s, x(t), I^{\alpha} x(t)\right)\right) d s \\
& \left.\left.\leq \frac{1}{\Gamma(\alpha)} \int_{0}^{t_{1}}\left(t_{1}-s\right)^{\alpha-1} g\left(s, x(t), I^{\alpha} x(t)\right)\right) d s-\frac{1}{\Gamma(\alpha)} \int_{0}^{t_{1}}\left(t_{2}-s\right)^{\alpha-1} g\left(s, x(t), I^{\alpha} x(t)\right)\right) d s \\
& \left.\left.+\frac{1}{\Gamma(\alpha)} \int_{0}^{t_{1}}\left(t_{2}-s\right)^{\alpha-1} g\left(s, x(t), I^{\alpha} x(t)\right)\right) d s-\frac{1}{\Gamma(\alpha)} \int_{0}^{t_{2}}\left(t_{2}-s\right)^{\alpha-1} g\left(s, x(t), I^{\alpha} x(t)\right)\right) d s \\
& \left.\left.\leq \frac{1}{\Gamma(\alpha)} \int_{0}^{t_{1}}\left(t_{1}-s\right)^{\alpha-1} g\left(s, x(t), I^{\alpha} x(t)\right)\right) d s-\int_{0}^{t_{1}}\left(t_{2}-s\right)^{\alpha-1} g\left(s, x(t), I^{\alpha} x(t)\right)\right) d s \\
& \left.\left.+\frac{1}{\Gamma(\alpha)} \int_{0}^{t_{1}}\left(t_{2}-s\right)^{\alpha-1} g\left(s, x(t), I^{\alpha} x(t)\right)\right) d s-\int_{0}^{t_{2}}\left(t_{2}-s\right)^{\alpha-1} g\left(s, x(t), I^{\alpha} x(t)\right)\right) d s \\
& \left.\leq \frac{\|h\|}{\Gamma(\alpha)} \int_{0}^{t_{1}}\left(t_{1}-s\right)^{\alpha-1}-\left(t_{2}-s\right)^{\alpha-1}\right) d s+\int_{t_{1}}^{t_{2}}\left(t_{2}-s\right)^{\alpha-1} d s \\
& \leq \frac{\|h\|}{\Gamma(\alpha)}\left(t_{1}^{\alpha}-t_{2}^{\alpha}+\left(t_{1}-t_{2}\right)^{\alpha} .\right.
\end{aligned}
$$

Since $t^{\alpha}$ is uniformly continues on $J$, so for any $\varepsilon>0$ there exist $\delta_{2}>0$ such that $\left|\left(t_{1}-t_{2}\right)\right|<\delta_{2}$ we have

$$
\mid\left(t_{1}^{\alpha}-t_{2}^{\alpha} \mid<\frac{\Gamma(\alpha+1)}{2\|h\|} \varepsilon\right.
$$

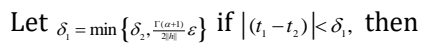

$$
\left|B x\left(t_{1}\right)-B x\left(t_{2}\right)\right|<\frac{\|h\|}{\Gamma(\alpha+1)}\left(\frac{\Gamma(\alpha+1)}{2\|h\|} \varepsilon+\frac{\Gamma(\alpha+1)}{2\|h\|} \varepsilon\right)=\varepsilon .
$$

By Arzel $a$-Ascoli theorem, $S$ is compact and continuous operator on 
$W$ which implies that $S(W)$ is compact subset of $X$. Now, we show that the last condition of Theorem 1.10 is also hold and inview of which $B(s)$ is equi-continuous and thus $B(s)$ is completely continuous on $S$. Let $x \in X$ and $y \in S$ such that $x=A x+B y$ by Assumption $H_{2}$ and $H_{3}$, we have

$$
\begin{aligned}
& |x(t)| \leq|A x(t)|+|B y(t)| \\
& \left|f(t, x(t))+t^{n-1}\left[\frac{\delta[f(\eta, x(\eta))-f(1, x(1))}{\left(1-\eta^{n-1}\right)}\right]+\right| I^{a} g\left(t, y(t), I^{\alpha} y(t)\right) \\
& +t^{n-1}\left[\frac{\delta\left[I^{a} g\left(\eta, y(\eta), I^{a}\right) y(\eta)\right]-I^{a} g\left(1, y(1), I^{a} y(1)\right)}{\left(1-\eta^{-1}\right)}\right] . \\
& \left.\Rightarrow|x(t)| \leq L+F_{0}+\frac{1}{\Gamma(\alpha)} \mid \int_{0}^{\prime}(t-s)^{\alpha-1} g\left(s, x(t), I^{a} x(t)\right)\right) d s+\delta[f(\eta, x(\eta))-f(1, x(1)) \mid \\
& \quad+\delta\left[I^{a} g\left(\eta, y(\eta), I^{a}\right) y(\eta)\right]-I^{a} g\left(1, y(1), I^{\alpha} y(1)\right) \mid \\
& \quad \leq L+F_{0}+\frac{\|h\|}{\Gamma(\alpha)}+\frac{\delta\|h\|}{\Gamma(\alpha)}+\frac{\|h\|}{\Gamma(\alpha)}+L+\frac{\delta L}{2} \\
& =2 L+F_{0}+\frac{2\|h\|}{\Gamma(\alpha)}+\frac{\delta\|h\|}{\Gamma(\alpha)}+\frac{\delta L}{2} \leq N .
\end{aligned}
$$

Which implies that $x \in S$. So, the assumption $\left(C_{3}\right)$ of Theorem 1 has been hold. Thus, all condition of theorem is satisfied hence the operator $T(x, y)=A x+B y$ has a coupled fixed point on $\tilde{S}$. Therefore, the system of integral equations (1) has a solution defined on $J$.

\section{EXAMPLE}

Example 2.1 Consider the following Hybrid coupled system of FDEs by taking $n>3$

$$
\left\{\begin{array}{l}
D^{\prime s}\left[x(t)-\left(e^{-t}+\frac{\frac{(t+1)}{4}|x(t)|}{40+|x(t)|}\right)\right]=\frac{t}{2}+\frac{e^{-t}}{2}\left[\sin |y(t)|+\sin \left|I^{\prime s} y(t)\right|\right], t \in[0,1], \\
D^{\prime \prime}\left[y(t)-\left(e^{-t}+\frac{(t+1)|y(t)|}{40+|y(t)|}\right)\right]=\frac{t}{2}+\frac{e^{-t}}{2}\left[\sin |x(t)|+\sin \left|I^{\prime s} x(t)\right|\right], t \in[0,1], \\
x(0)=x^{\prime}(0)=0, x(1)=\frac{1}{3} x\left(\frac{1}{3}\right), y(0)=y^{\prime}(0)=0, y(1)=\frac{1}{3} y\left(\frac{1}{3}\right) .
\end{array}\right.
$$

From (2.1), we have

$$
\begin{aligned}
& f(t, x(t))=e^{-t}+\frac{\frac{(t+1)}{4}|x(t)|}{40+|x(t)|}, \\
& g(t, x(t), y(t))=\frac{t}{2}+\frac{e^{-t}}{2}\left[\sin |y(t)|+\sin \left|I^{1.5} y(t)\right|\right] \leq \frac{t}{2} .
\end{aligned}
$$

Now it is easy to fine

$$
\begin{gathered}
L=\frac{1}{2}, M=40, F_{0}=1, ? h ?=\frac{1}{4}, \delta=\frac{1}{3} \\
2 L+F_{0}+\frac{2\|h\|}{\Gamma(\alpha)}+\frac{\delta\|h\|}{\Gamma(\alpha)}+\frac{\delta L}{2}=2+\frac{4}{3 \sqrt{\pi}}+\frac{1}{12} \leq 3=N
\end{gathered}
$$

Hence all the conditions of Theorem 1.10 are satisfied, which show that the FHDEs system has a solution in $\tilde{S}=\{x \in X:\|x\| \leq 3\}$.

\section{REFERENCES}

[1] Burton, T.A. 1998. A fixed-point theorem of Krasnoseiski. Applied Mathematics Letters, 11 (1), 85-88.

[2] Basiri, T., Vaezpour, S.M., and Park, C. 2016. Acoupled fixed point theorems and application to fractional hybrid diffrential problem. Fixed Point Theory and Applications, 2016, Article ID 23.

[3] Caputo, M. 1967. Linear Models of dissipation whose $Q$ is almost frequency independent. Geophysical Journal International, 13 (5), 529539.

[4] Dhage, B. C. 2004. A fixed-point theorem in Banach algebras involving three operators with applications. Kyungpook Mathematical Journal, 44 (2004), 145-155.

[5] Dhage, B. C., Lakshmikantham, V. 2010. Basic results on hybrid differential equations. Nonlinear Analysis, 4 (3), 414-424.
[6] Dhage, B.C. 2004. A nonlinear alternative in Banach algebras with applications to functional differential equations. Nonlinear functional analysis and applications, 8 (2004), 563-575.

[7] Dhage, B.C. 1999. Fixed point theorems in ordered Banach algebras and applications. Panamerican Mathematical Journal, 9, 93-102.

[8] Baleanu, D., Khan, H., Jafari, H., Khan, R. A., and Alipour, M. 2015. On existence results for solutions of coupled system of Hybrid boundary value problems with Hybrid conditions. Advances in Difference Equations, 318 (2015), 1-14

[9] Hilal, K., and Kajouni, A. 2015. Boundary value problem for hybrid differential equations with fractional order. Advances in Difference Equations, 183 (2015), 1-19.

[10] Herzallah, M. A. E., and Baleanu, D. 2014. On Fractional Order Hybrid Differential Equations. Abstract and Applied Analysis, 2014 (2014), 7 pages.

[11] Su, X. 2009. Boundary value problem for a coupled system of nonlinear fractional differential equations. Applied Mathematics Letters, 22 (1), 64-69.

[12] Shah, K., and Khan, R. A. 2015. Existence and uniqueness of positive solutions to a coupled system of nonlinear fractional order differential equations with anti-periodic boundary conditions. Journal of Difference Equations and Applications, 7 (2), 245-262.

[13] Shah, K., Khalil, H., and Khan, R. A. 2015. Investigation of positive solution to a coupled system of impulsive boundary value problems for nonlinear fractional order differential equations. Chaos, Solitons and Fractals, 77 (2015), 240-246.

[14] Dhage, B.C., Karande, B.D. 2005. First order integro-differentia equations in Banach algebras involving Caratheodory and discontinuous nonlinearities. Electronic Journal of Qualitative Theory of Differential Equations, 21, 1-16.

[15] Dhage, B.C., O'Regan, B.D. 2000. A fixed-point theorem in Banach algebras with applications to functional integral equations. Functional Differential Equations, 7 (3-4), 259-267.

[17] Dhage, B.C. 1994. On $\alpha$-condensing mappings in Banach algebras. Journal of Mathematical Study, 63, 146-152.

[18] Dhage, B.C., Lakshmikantham, V. 2010. Basic results on hybrid differential equations. Nonlinear Analysis: Hybrid Systems, 4, 414-424.

[19] Dhage, B.C. 2004. A nonlinear alternative in Banach algebras with applications to functional differential equations. Nonlinear functional analysis and applications, 8, 563-575.

[20] Dhage, B.C. 1999. Fixed point theorems in ordered Banach algebras and applications. Panamerican mathematical journal, 9, 93-102.

[22] Kilbas, A. A., Marichev, O.I., and Samko, S. G. 1993. Fractional Integrals and Derivatives (Theory and Applications), Gordon and Breach, Switzerland.

[23] Miller, K. S., and Ross, B. 1993. An Introduction to the Fractional Calculus and Fractional Differential Equations, Wiley, New York.

[24] Podlubny, I. 1999. Fractional Differential Equations, Mathematics in Science and Engineering, Academic Press, New York.

[25] Hilfer, R. 2000. Applications of Fractional Calculus in Physics, World Scientific, Singapore.

[26] Kilbas, A. A., Srivastava, H. M., and Trujillo, J.J. 2006. Theory and Applications of Fractional Differential Equations, North-Holland athematics Studies, 204, Elsevier, Amsterdam.

[27] El-Shahed, M., and Nieto, J. J. 2010. Nontrivial solutions for a nonlinear multi-point boundary value problem of fractional order. Computers and Mathematics with Applications, 59 (11), 3438-3443.

[28] Li, C. F., Luo, X. N., and Zhou, Y. 2010. Existence of positive solutions of the boundary value problem for nonlinear fractional differential equations. Computers and Mathematics with Applications, 59 (3), 1363-1375. 
[29] Rehman, M., and Khan, R. A. 2010. Existence and uniqueness of solutions for multi-point boundary value problems for fractional differential equations. Applied Mathematics Letters, 23 (9), 1038-1044.

[30] Zhong, W., and Lin, W. 2010. Nonlocal and multiple-point boundary value problem for fractional differential equations. Computers and Mathematics with Applications, 59 (3), 1345-1351.

[31] El-Shahed, M., and Shammakh, W.M. 2011. Existence of positive solutions for m-point boundary value problem for nonlinear fractional differential equation. Abstract and Applied Analysis, Article ID 986575, 20.

[32] Cui, Z., Yu, P., and Mao, Z. 2012. Existence of solutions for nonlocal boundary value problems of nonlinear fractional differential equations. Advance Dynamic Systems and Applications, 7 (1), 31-40.

[33] Khan, R. A. 2013. Three-point boundary value problems for higher order nonlinear fractional differential equations. Journal of Applied Mathematics and Informatics, 31 (1-2), 221-228.

[34] EL-Sayed, A. M. A., and Bin-Taher, E.0.2013. Positive solutions for a nonlocal multi-point boundary-value problem of fractional and second order. Electronic Journal of Differential Equations, 64, 1-8.
[35] Dhage, B. C., and Lakshmikantham, V. 2010. Basic results on hybrid differential equations. Nonlinear Analysis: Hybrid Systems, 4 (3), 414 424.

[36] Dhage, B., and Jadhav, N. 2013. Basic results in the theory of hybrid differential equations with linear perturbations of second type. Tamkang Journal of Mathematics, 44 (2), 171-186.

[37] Ammi, M. R., El Kinani, E., and Torres, D. F. 2012. Existence and uniqueness of solutions to functional integro-differential fractional equations. Electronic Journal of Differential Equations, 2012 (103), 1-9.

[38] Lu, H., Sun, S., Yang, D., and Teng, H. 2013. Theoryof fractional hybrid differential equations with linear perturbations of second type. Boundary Value Problems, 2013 (23).

[39] Mohamed, A. E., Herzallah1, and Baleanu, D. 2014. On Fractional Order Hybrid. Differential Equations Abstract and Applied Analysis Volume 2014, Article ID 389386, 7 pages.

[40] Gafiychuk, V., Datsko, B., Meleshko, V., Blackmore, D. 2009. Analysis of the solutions of coupled nonlinear fractional reaction-diffusion equations. Chaos Solitons Fractals 41, 1095-1104.

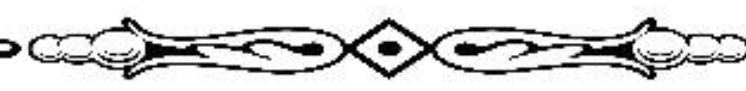

